\title{
INTRODUCCIÓN DEL SISTEMA DE ADUERTENCIAS AL CONSUMIDOR BASADO EN OCTÓGONOS Y EL CONSUMO DE ALIMENTOS PROCESADOS EXCESIVOS EN NUTRIENTES
}

INTRODUCTION OF THE OCTOGON-BASED CONSUMER WARNING SYSTEM AND CONSUMPTION OF EXCESSIVE PROCESSED FOOD IN NUTRIENTS

Muriel Alférez Murias ${ }^{1}$

Sebastián Alférez Murias²

Raul Cartagena cutipa ${ }^{3}$

Información del artículo:

Recibido: 10/0/2019

Aceptado: 01/05/2020

${ }^{1,2}$ Ingeniero Agroindustrial., E-mail: ${ }^{1}$ murielalferez@gmail.com, ${ }^{2}$ salferezmurias@gmail.com

${ }^{3}$ Docente en la Facultad de Ingeniería, Universidad Privada de Tacna.

E-mail: raulcart@hotmail.com

Vol. 2, No 1.

INGENIERÍA

Enero - Junio del 2020. 


\section{Resumen}

La investigación tuvo como objetivo determinar efectos potenciales en la introducción del sistema de advertencias al consumidor basado en "octógonos" en el consumo de alimentos procesados excesivos en nutrientes. La investigación es de tipo no experimental y transversal.se uso la encuesta como técnica, aplicando un cuestionario a población de 15 a 64 años provenientes de los cinco distritos más poblados de la provincia de Tacna: Coronel Gregorio Albarracín, Tacna, Alto de la Alianza, Ciudad Nueva y Pocollay. Para el procesamiento y analistas de datos se aplicó el procedimiento de "Tablas cruzadas" y "Chi - Cuadrado". Los resultados indican que los "Panes y productos horneados" son los alimentos excesivos en nutrientes que más consume la población tacneña. La mayor parte de los encuestados desconoce la Ley de Promoción de la Alimentación Saludable para Niños, Niñas y Adolescentes $N^{\circ} 30021$, por lo que un porcentaje elevado de esta asegura que generará un impacto positivo en la población, estando en "Total acuerdo" respecto al uso de rótulos octogonales y entendiendo mejor el etiquetado de octógonos. Se concluye que el sistema de advertencias al consumidor basado en octógonos genera efectos, tanto positivos como negativos en los hábitos de consumo de alimentos procesados excesivos en nutrientes críticos.

Palabras Claves: Alimentos excesivos en nutrientes; Alimentación Saludable; Octógonos; nutricionales.

\section{Abstract}

The research aimed to determine potential effects on the introduction of the "octagon" based consumer warning system on the consumption of processed foods that are excessive in nutrients. The research is non-experimental and cross-sectional. The survey was used as a technique, applying a questionnaire to the population aged 15 to 64 from the five most populated districts of the province of Tacna: Colonel Gregorio Albarracín, Tacna, Alto de la Alianza , Ciudad Nueva and Pocollay. For the processing and data analysts the procedure of "Cross Tables" and "Chi - Square" was applied. The results indicate that "Breads and baked goods" are the excessive nutrients that the Tacna population consumes the most. Most of the respondents are unaware of the Law for the Promotion of Healthy Food for Boys, Girls and Adolescents No. 30021, so a high percentage of this ensures that it will generate a positive impact on the population, being in "Total agreement" regarding to the use of octagonal labels and better understanding of octagon labeling. It is concluded that the octagon-based consumer warning system generates effects, both positive and negative, on the consumption habits of processed foods excessive in critical nutrients.

Keywords: Excessive food in nutrients; Healthy nutrition; Nutritional; Octagons. 


\section{Introducción}

El término alimentación, según la Real Academia Española, es la acción de alimentar o alimentarse. Esta es una cotidiana acción que las personas realizan con el fin de proporcionar sustancias que tengan nutrientes necesarios para el desarrollo del organismo humano. Dicho esto, las personas tenemos la capacidad de decidir y poder tomar las decisiones más convenientes para nuestra vida y futuro. Pero hay decisiones que se toman en el día a día, y una de estas es la alimentación, es decir que comemos. Es claro que sea por desconocimiento o costumbre, algunos o muchos de los alimentos que la población ingiere diariamente hacen daño. En los últimos tiempos, la población ha optado progresivamente por alimentos sin procesar sustituyéndolos por alimentos procesados de fácil preparación, no obstante otra parte de la población asume la alimentación "vegana" aunque aún en la población no es significativa. Debido a estos cambios, la salud de la población se ve afectada seriamente, manifestando índices de sobrepeso y obesidad que se incrementan, estudios en esa dirección señalan como problemática a nivel mundial (Organización Mundial de la Salud, 2016., Pajuelo-Ramirez, 2017., González, 2018), ello conlleva a enfermedades crónicas no trasmisibles, y un consecuente sobredimensionamiento en los gastos de salud pública, por ello el estado peruano, mediante la dación de la Ley de Promoción de la Alimentación Saludable para Niños, Niñas y Adolescentes $N^{\circ} 30021$, tiene por objetivo la promoción y publicidad, la información y otras prácticas relacionadas con los alimentos y bebidas no alcohólicas dirigida a los niños, niñas y adolescentes, para reducir las enfermedades relacionadas con el sobrepeso, obesidad y crónicas, causadas por alimentos que tienen componentes cuyo consumo debe ser limitado, y son excesivos como son el azúcar, el sodio, las grasas saturadas y las grasas trans.

Es conocido también que el consumo inconsciente de alimentos críticos en nutrientes se debe a los defectos en el etiquetado de los alimentos que no advierten adecuadamente al consumidor, como la información nutricional en la parte posterior, letras muy pequeñas y la información muy técnica, esto hace que el consumidor pierda interés por la información del producto y consuma sin ningún reparo; en esa dirección DATUM Internacional (2018), indica que la mayor parte de la población peruana se informa "Poco o nada" de los ingredientes que constituyen los productos que van a consumir.

\section{Objetivos}

En esa dirección el estudio indaga en los efectos potenciales en la introducción del sistema de advertencias al consumidor basado en octógonos en el etiquetado frontal de alimentos procesados excesivos en nutrientes, tomando en consideración conocer si el consumidor es consciente y conoce las advertencias de los octógonos nutricionales, así mismo las implicancias en la selección de los alimentos.

\section{Metodología}

La investigación es de tipo no experimental y transversal, en razón que variables permiten medir Implicancias potenciales de la introducción del sistema de advertencias al consumidor basado en octógonos en el etiquetado frontal de alimentos procesados excesivos en nutrientes. Tal como afirman Hernández, Fernández y Baptista (2010), los diseños transaccionales descriptivos tienen como objetivo indagar la incidencia de las modalidades o niveles de una o más variables en una población. Por tanto las

Ingeniería Investiga Vol. 2 (1) Enero - diciembre 2020 
variables a medir fueron; Implicancias potenciales de la introducción del sistema de advertencias al consumidor basado en octógonos y Etiquetado frontal de alimentos procesados excesivos en nutrientes.

La muestra de estudio estuvo conformada por consumidores de la provincia de Tacna, que se compone de 5 distritos, el muestreo fue de carácter probabilístico, con el objetivo que sea representativa de la población. Para los criterios de exclusión e inclusión se tomaron en cuenta consumidores que podrían responder sin segar la información deseada, por tanto, la muestra de estudio se compone de consumidores de 15 a 64 años (a más) de edad, así mismo se limita a la población urbana. Según la información obtenida de INEI (2017), la población determinada según "La Encuesta de Población y Vivienda efectuada el año 2017", son 220479 pobladores que se distribuyen en los distintos distritos de la provincia de Tacna, de los cuales se tomó los distritos más representativos: Coronel Gregorio Albarracín con 80801 habitantes, Tacna con 73953 habitantes, Alto de la alianza con 26914 , Ciudad Nueva con 24144 habitantes y Pocollay con 14667 habitantes. Por lo tanto, la población para la investigación es de 220479 habitantes entre 15 a 64 años (a más). Para calcular la muestra para la investigación se calculó empleando la fórmula reportada por Hernández, Fernández y Baptista (2010) resultando 384 personas que se distribuyen proporcionalmente en La tabla 2 refleja la distribución de los estudiantes que constituyen la muestra. Distrito de Tacna 33,54 \%, Distrito de Pocollay 6,67 \%, Ciudad Nueva 10,95 \%, Alto del Alianza 12.22 \% y Cnel. Gregorio Albarracín 36,64 \%

La información recogida se analizó usando software estadístico IBM SPSS Estadistics 24, para el análisis de datos cuantitativos. En primera instancia se realizó con un análisis exploratorio de datos, con la finalidad de representar la población de la cual proceden los datos muéstrales y luego fueron representados en gráficos estadísticos que permiten explorar la distribución identificando las características. Este análisis se realizó sobre todos los casos de forma conjunta y de forma separada por grupos. Posteriormente, se empleó la inferencia estadística, para hacer predicciones y estimaciones. Para identificar relaciones de dependencia entre variables se utilizó un contraste estadístico basado en el estadístico Chi - cuadrado y la prueba de R de Pearson.

\section{Resultados}

\subsection{Tipo de alimentos excesivos en nutrientes que consume la población tacneña}

Se determinó que el alimento que más consume la población tacneña diariamente, son (los) Panes y Productos Horneados con un $81,1 \%$, no muy alejado de otro producto elaborado a base de trigo, que son las Pizzas y Pastas listas para Comer con un 59,7 \%. Entre los productos lacteos, el Queso es el producto que los encuestados mas consumen con un $76,4 \%$, con $17,9 \%$ se encuentra los Yogures y Bebidas lacteas, como: leche chocolatada, yogurth griego, leche sin lactosa, entre otros. Los resultados se representan en la figura 1.

\subsection{Actitud del consumidor frente al etiquetado de alimentos con sistema de octágonos}

Según la figura 2, el 28,1 \% de encuestados A veces se fija en la información de un producto o bebida al momento de comprar. No muy distante y con una cifra alarmante, el $26,2 \%$ Nunca se fija en tal información. Siguiendo con el análisis, el 20,5 \% de los encuestados manifiesta estar Casi siempre informado al momento de adquirir un producto procesado o bebida. El $15,8 \%$ de los encuestados, afirma que Casi nunca se fijan en la información del producto que están adquiriendo. La afirmación respecto a que el encuestado Siempre está informado al momento de adquirir un alimento procesado es 
$9,4 \%$. Finalmente, el $26,2 \%$ de los encuestados afirma que Nunca se fija en la información al momento de comprar.

Figura 1

Representación de alimentos excesivos en nutrientes que consume la población tacneña

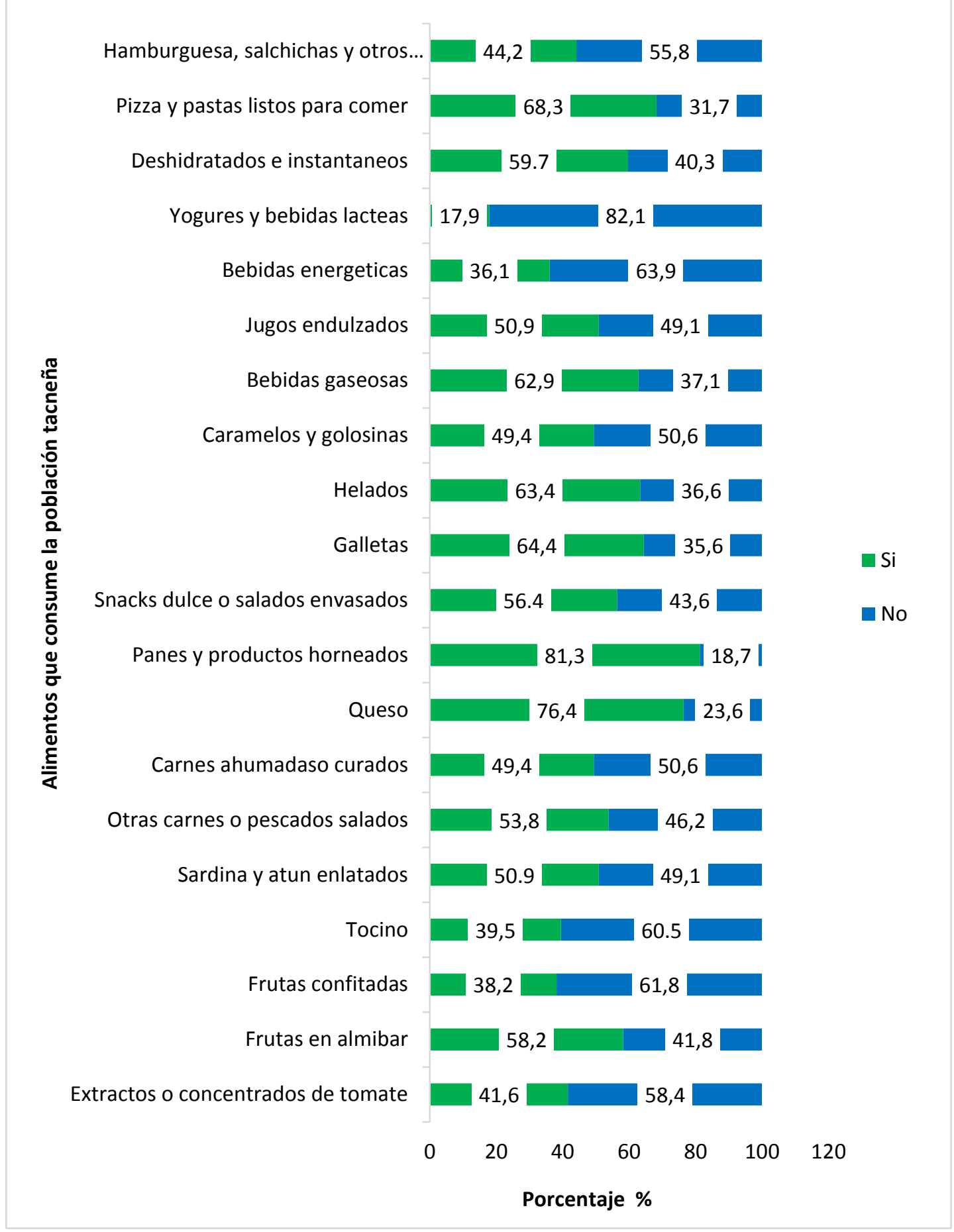

Nota. Panes y Productos Horneados, los más consumidos. Adaptado de encuesta Alimentación saludable y las implicancias de la normativa en el consumidor - 2019. 


\section{Figura 2}

Representación en la que el encuestado se fija en la información nutricional al momento de comprar.

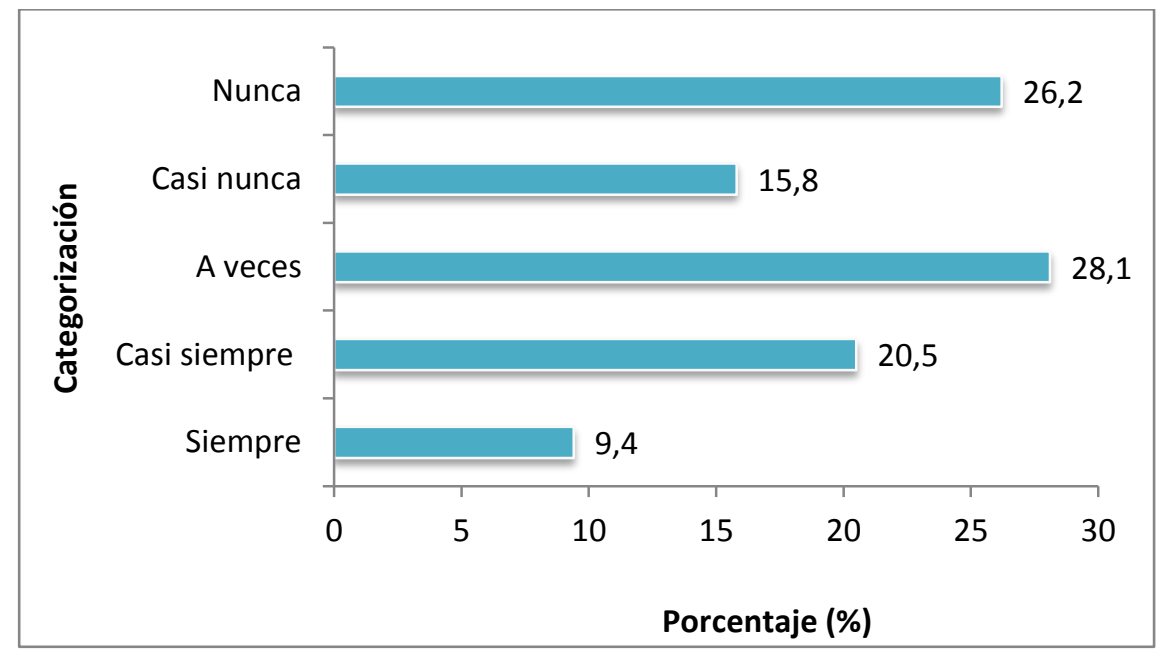

Nota. Adaptado de encuesta Alimentación saludable y las implicancias de la normativa en el consumidor - 2019.

En la figura 3 se describe sobre el tipo de información que el encuestado se fija en las etiquetas de los alimentos y bebidas, el envase de un alimento procesado se encuentra información de utilidad para el consumidor, siendo la Fecha de vencimiento la información que los encuestados se fijan principalmente, esta equivale a un 30,9\%; en segundo lugar el Nombre del alimento es la información que el encuestado tiene en cuenta, esto representa al 22,7 \%. El 16,9\% asegura que el Contenido neto es lo que toman en cuenta para elegir al momento de comprar; le sigue el 16,4 \% que es la Lista de ingrediente. Finalmente, lo que menos observa el encuestado al adquirir un producto son Las instrucciones para su preparación, Registro sanitario y Nombre y dirección del fabricante con un 13,2 \%.

Los atributos de un producto son las características que lo conforman, estas aportan alguna utilidad al consumidor. Tal como se puede aprecio en la figura 4, el Precio con el $36 \%$, es el atributo que se considera el más importante al momento de adquirir un alimento procesado; Seguido por el Tamaño del producto con un 16,9 \%. No muy distante, encontramos que la Marca equivale al 15,7 \%, atributo que el encuestado toma en cuenta. El Valor nutricional representa un 14,4 \%, seguido por la Presentación del producto con 9,2\%. Los atributos que menos se toman en cuenta al momento de adquirir un producto, vienen a ser la Seguridad del producto y Facilidad de preparación o consumo, con $4,6 \%$ y $0,2 \%$ respectivamente.

En la figura 5 refleja un resultado Positivo a la aplicación de la Ley de Alimentación Saludable, donde un 83, $1 \%$ indica que su aplicación es positiva, otro $15 \%$ de los encuestados no saben de su aplicación, Finalmente, 4 personas que equivalen al $1 \%$, considera que su aplicación es negativa. 


\section{Figura 3}

Representación del tipo de información que el encuestado se fija en las etiquetas de los alimentos y bebidas

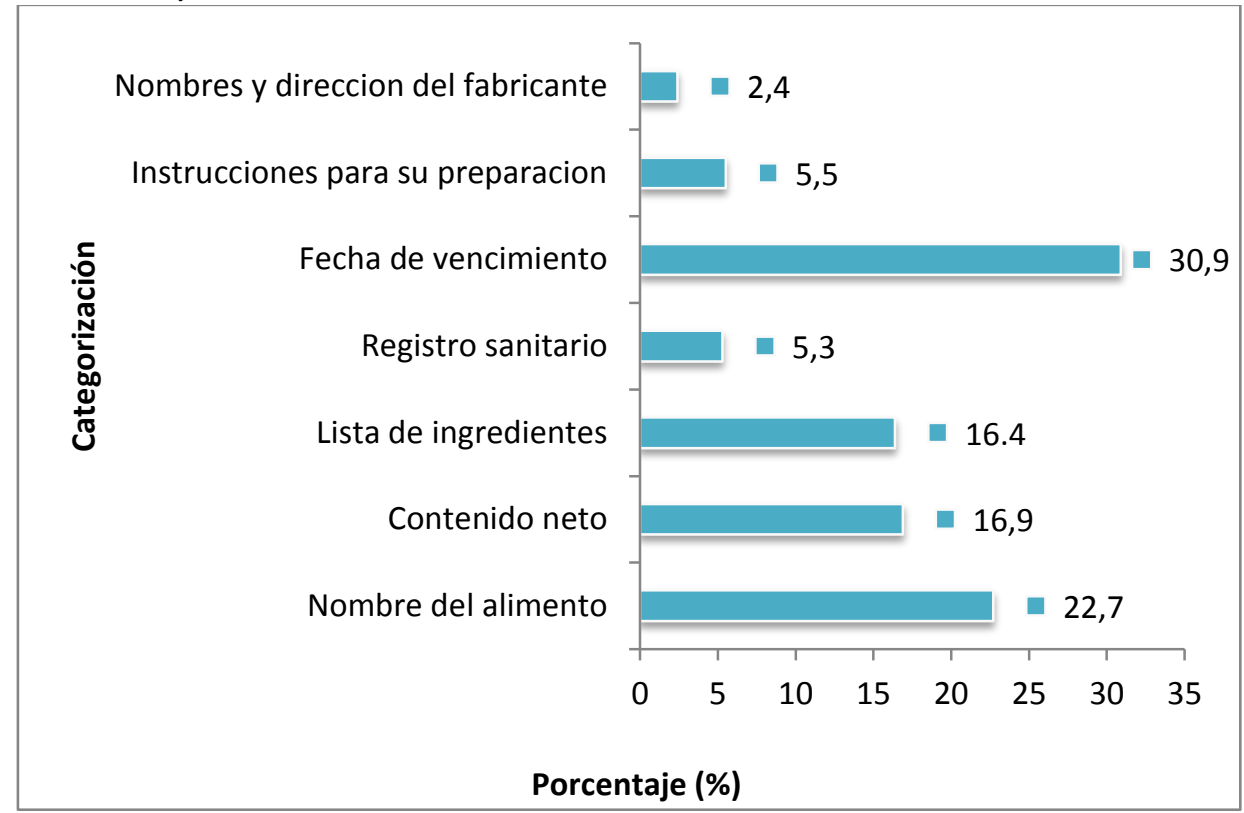

Nota. Adaptado de encuesta Alimentación saludable y las implicancias de la normativa en el consumidor - 2019.

\section{Figura 4}

Representación en la que el consumidor considera los son los atributos más importantes de un producto.

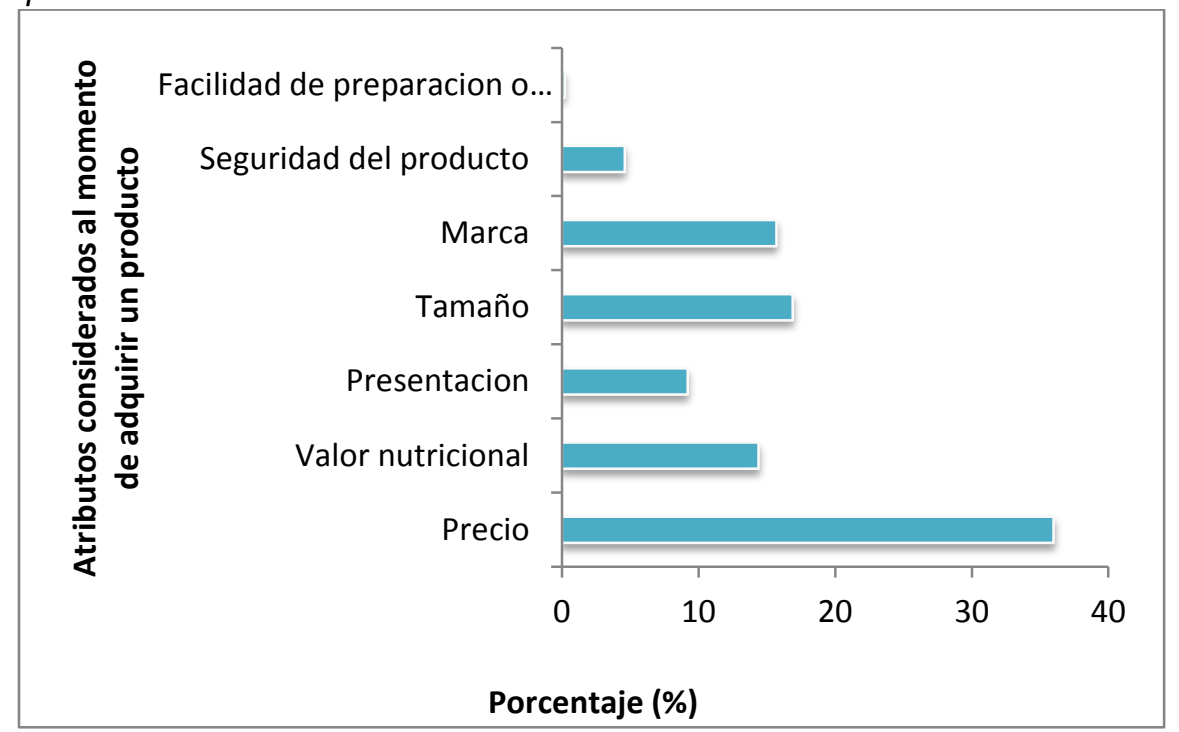

Nota. Adaptado de encuesta Alimentación saludable y las implicancias de la normativa en el consumidor - 2019. 


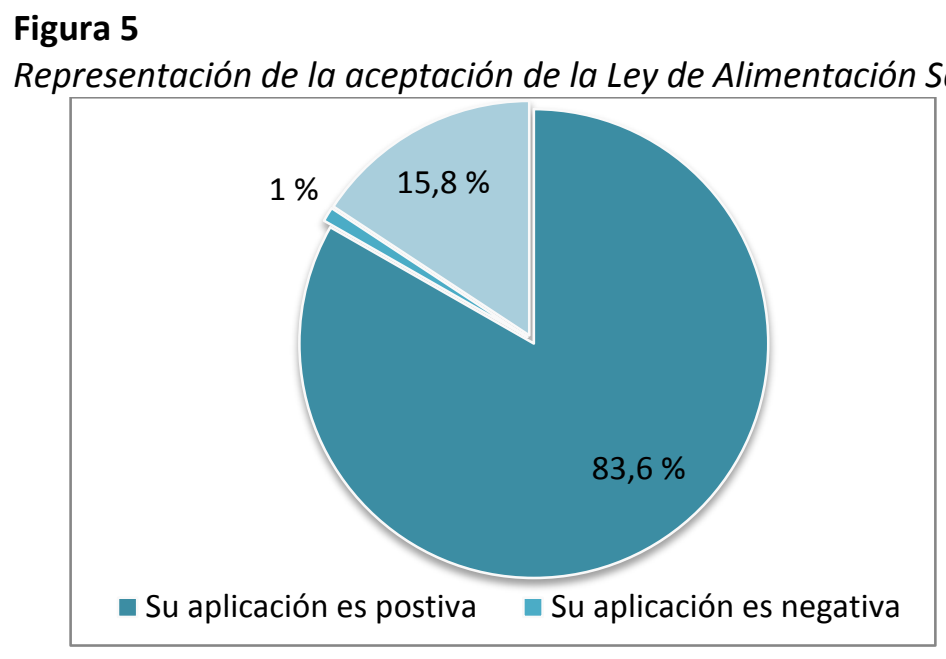

Nota. Adaptado de encuesta Alimentación saludable y las implicancias de la normativa en el consumidor - 2019

4.3 Relación existente entre los octágonos con las decisiones informadas en la selección de productos que son considerados saludables

Se identificó que el problema es la falta de información sobre la Ley de Alimentación Saludable $\mathrm{N}^{\circ} 30021$, debido a que solo el $43,9 \%$ está enterado de esta, el 56,1 \% afirman no haber escuchado hablar sobre la ley de Alimentación Saludable, la figura 6 representa lo afirmado.

\section{Figura 6}

Representación sobre el conocimiento de la Ley de Alimentación Saludable Na 30021.

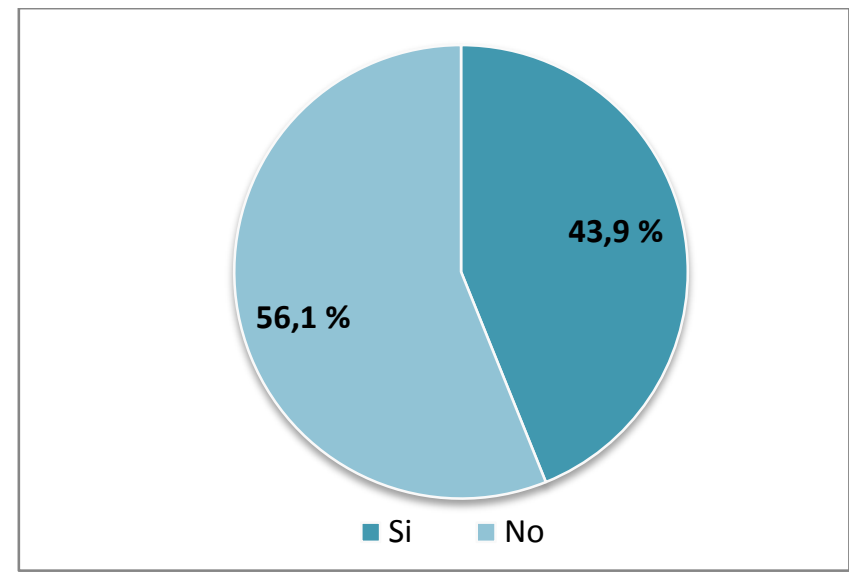

Nota. Adaptado de encuesta Alimentación saludable y las implicancias de la normativa en el consumidor - 2019

De acuerdo a la figura 7, el 39,2 \% de los encuestados afirman estar Totalmente de Acuerdo con que los alimentos envasados deban de tener un rotulo que adviertan a los usuarios sobre los altos de 
insumos. No muy distante, el 38,7 \% de los encuestados están De Acuerdo con el rotulado de la Ley de Alimentación Saludable.

\section{Figura 7}

Representación respecto a la aprobación de los rótulos que advierte a los encuestados sobre los altos niveles de insumos en los alimentos procesados

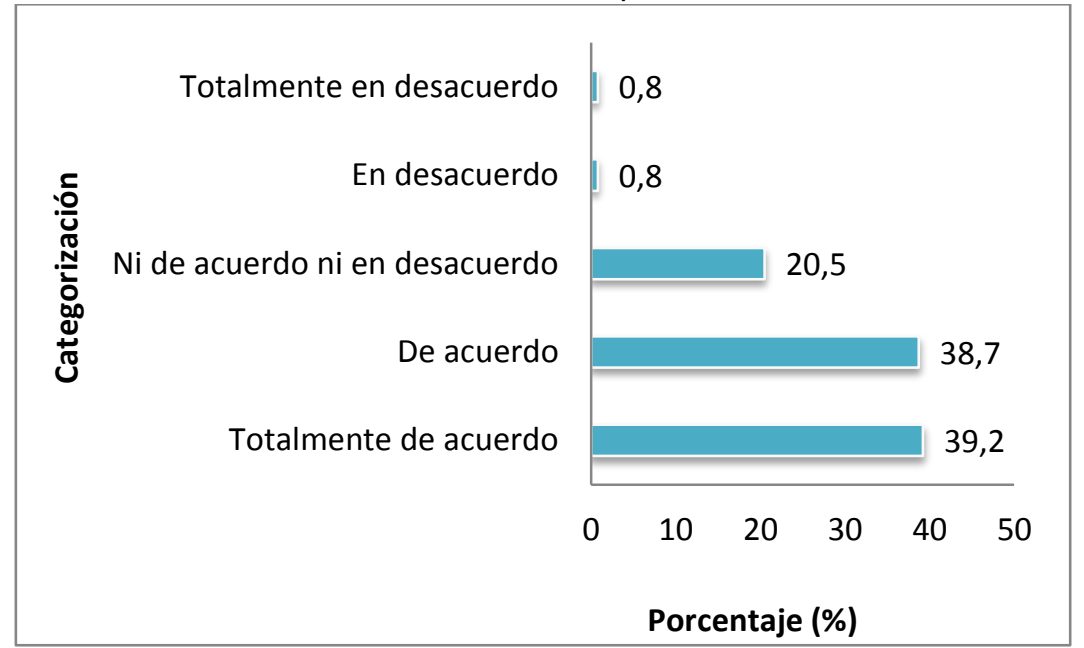

Nota. Adaptado de encuesta Alimentación saludable y las implicancias de la normativa en el consumidor - 2019.

\subsection{Relación entre el nivel de importancia que otorga el consumidor con que un producto que cuente con mensajes de alerta sobre los nutrientes críticos.}

El etiquetado nutricional de fácil comprensión es la propuesta para prevenir el sobre peso, obesidad $u$ otras condiciones que se vienen incrementando masivamente en estos tiempo. Su función es informar el contenido de lo que se consume, reflexionar y tomar la decisión que sea favorable para la salud. De esta manera, la figura 8 detalla que los encuestados consideran que hay octágonos más importantes que otros; el 34,3\% señala que el octágono de mayor importancia es el Alto en Azúcar. Seguido del 20,8 \% con Alto en Grasas Saturadas, el 20,7 \% que representa el octágono Alto en Sodio y $19,3 \%$ al octágono de Contenido de Grasas Trans. Por ultimo un 4,9 \% no considera Ninguno en particular.

La OMS respalda el uso de etiquetado octagonal, ya que considera que este es un sistema claro y conciso, a su vez proporciona información rápida y de fácil entendimiento. A demás advierte que el etiquetado tipo semáforo es confuso para el consumidor, que no tiene el conocimiento necesario para interpretar las cifras y porcentajes, limitando su derecho a la información y yendo en contra del objetivo central de la ley.

Tal como puede apreciarse en la figura 9, el 45,7 \% de los encuestados Entiende mejor el de Octágonos, seguido por el 30,9 \% que afirma que Entiende Ambos tipos de etiquetado. El 11,9\% Entiende mejor el de tipo Semáforo. Solo el $7 \%$ afirma No entender Ninguno de los etiquetados. Para concluir el análisis de esta figura, el 4,4 \% No Comenta sobre el tema. 
Figura 8

Representación acerca del octágono de mayor importancia para el encuestado

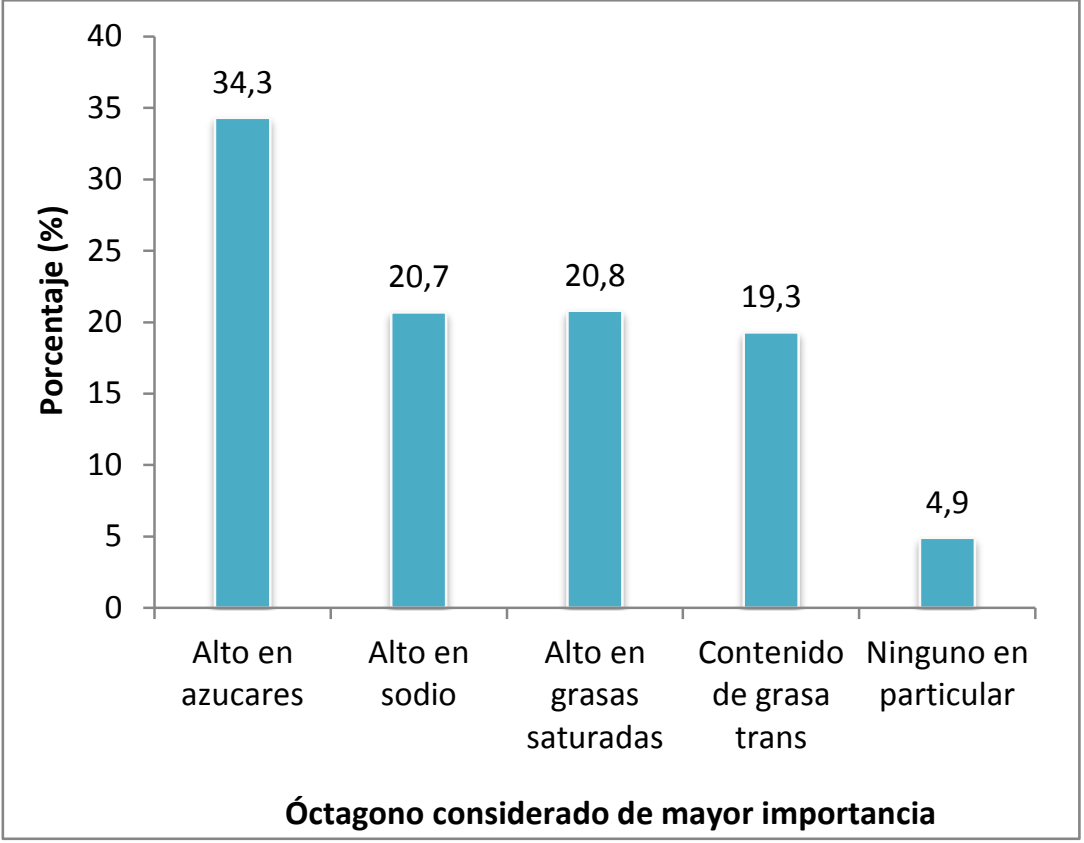

Nota. Adaptado de encuesta Alimentación saludable y las implicancias de la normativa en el consumidor - 2019.

\section{Figura 9}

Representación del octágono de mayor importancia para el encuestado

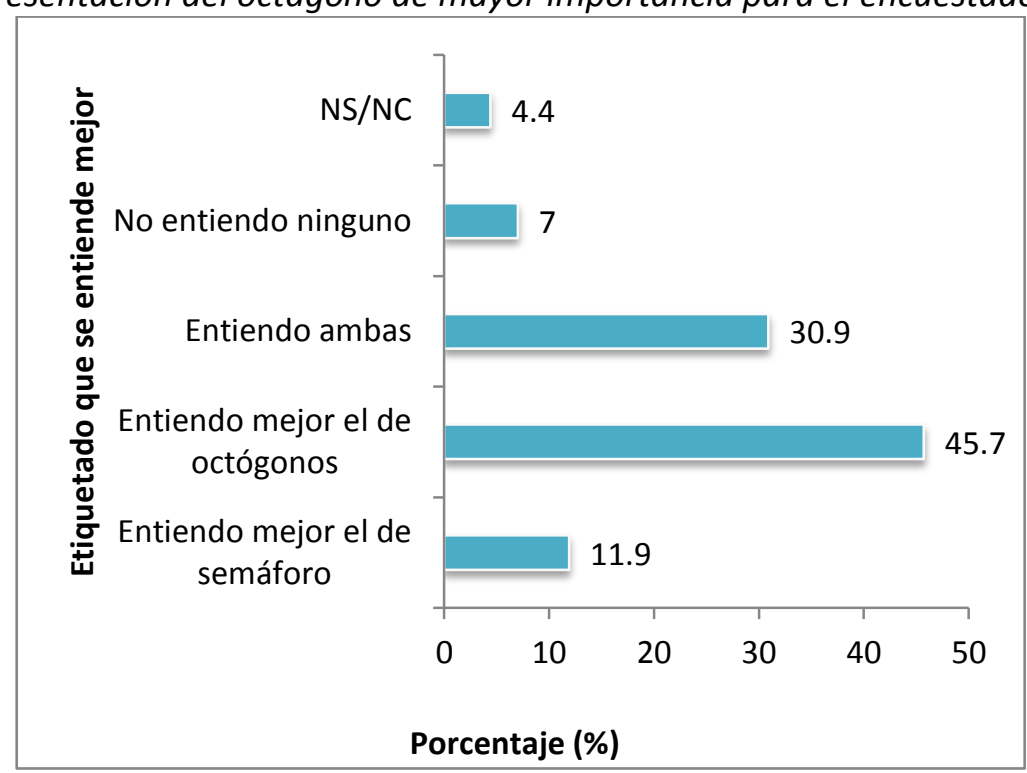

Nota. Adaptado de encuesta Alimentación saludable y las implicancias de la normativa en el consumidor - 2019. 
4.5 Productos que identifica el consumidor como críticos en contenido de nutrientes que exceden parámetros técnicos

Según los resultados, el encuestado relaciona más el octágono de "alto en sodio" con las Hamburguesas, salchichas y otros embutidos, Las Pizzas y las pastas listas para comer, el queso, carnes ahumadas, carnes curadas, otras carnes o pescados salados, sardina y atún enlatado y tocino. Así mismo otro grupo que identifica adecuadamente son los aquellos que son alto en azucares tales como; bebidas energéticas, jugos endulzados, bebidas gaseosas, caramelos y golosinas, helados, galletas, snacks, panes y productos horneados.

\subsection{Efectos sobre el consumo de productos del etiquetado en alimentos considerados críticos}

Con el objetivo de determinar si el etiquetado con octógonos modificará hábitos, reducirá y/o eliminará el consumo de algunos productos procesado, la figura 11 muestra que en general consumirá menos productos clasificados como críticos en nutrientes presentados en una lista, no obstante manifiestan que seguirán consumiendo de forma similar al actual los panes y productos horneados, bebidas gaseosas, quesos y helados.

4.7 Determinar la relación que existe entre la decisión de compra del consumidor y la implementación de sistema de octágonos

Según la figura 12, se observa que el 43,6 \% de los encuestados comprará Igual al ver los símbolos en el empaque de los productos. Además el 37,1 \% afirma que al ver los símbolos en los empaques Comprará Menos y el 16,6 \% Dejará de Comprar. Esta última decisión está considerada como positiva, debido a que el consumidor está tomando conciencia de que los productos procesados van a repercutir en la salud. Comprará menos es la actitud que debería ser la de todos los consumidores al momento de adquirir algún producto procesado, dicha información fue contrastado mediante prueba de chi cuadrado determinándose que existe una relación significativa $(p<0,005)$.

\subsection{Relación entre decisión de compra por parte del consumidor e implementación de octágonos.}

Los octágonos es un sistema correcto para el cuidado de la salud alimenticia. En la figura 13, el 61,8 \% de los encuestados no estaría dispuesto a pagar un poco más de dinero por la presencia de una certificación nutricional como la de los octágonos; sin embargo, el 37,9 \% de los encuestados siendo consciente de este tema si estaría dispuesto a pagar un poco más de dinero por la presencia del etiquetado de octágonos, ya que garantiza la composición respecto a los nutrientes críticos. 


\section{Figura 10}

Representación de octágonos del etiquetado nutricional que los encuestados relacionan con los alimentos que consumen

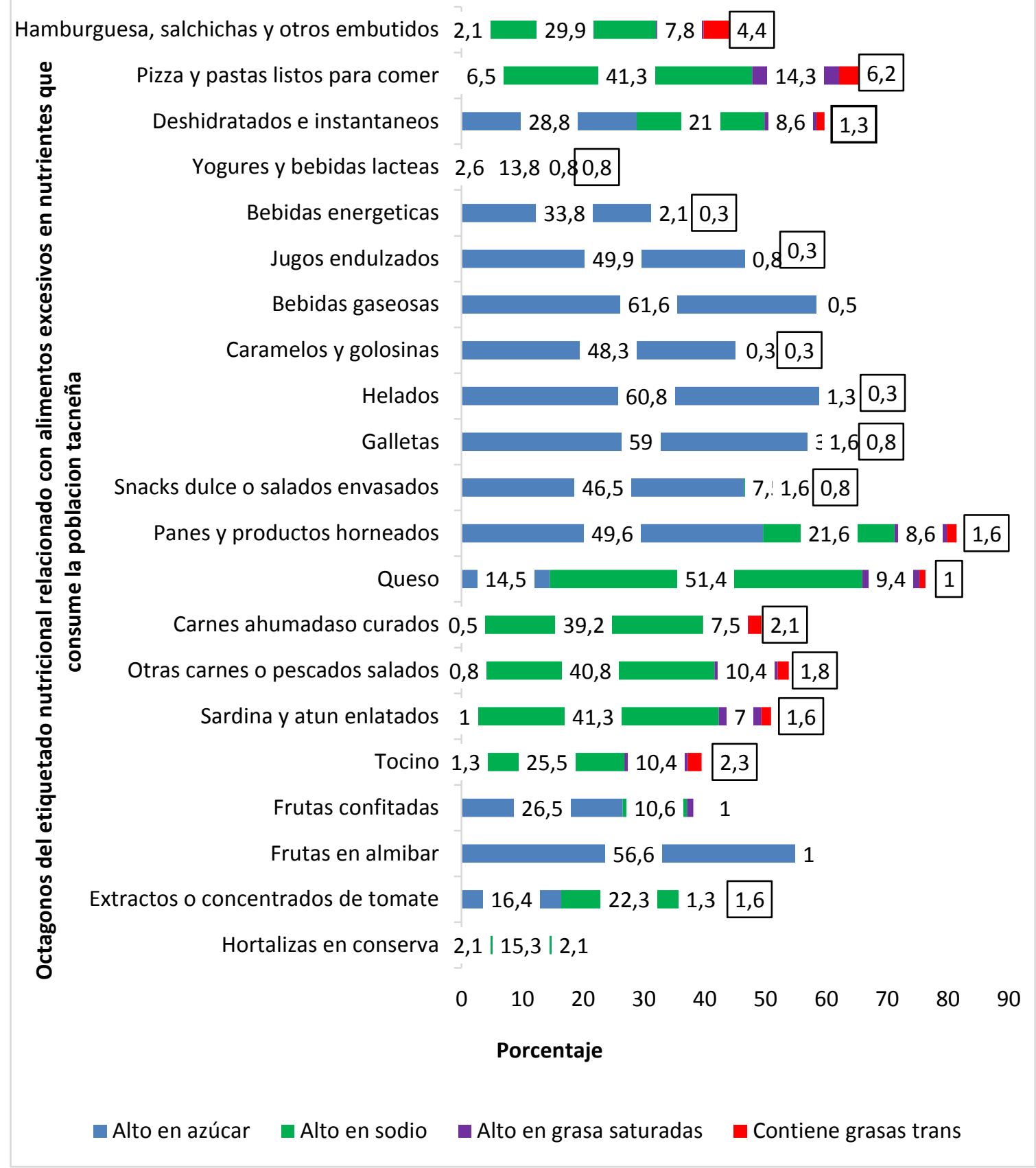

Nota. Adaptado de encuesta Alimentación saludable y las implicancias de la normativa en el consumidor -2019. 


\section{Figura 11}

Representación de la tendencia personal de consumo a futuro de los encuestados

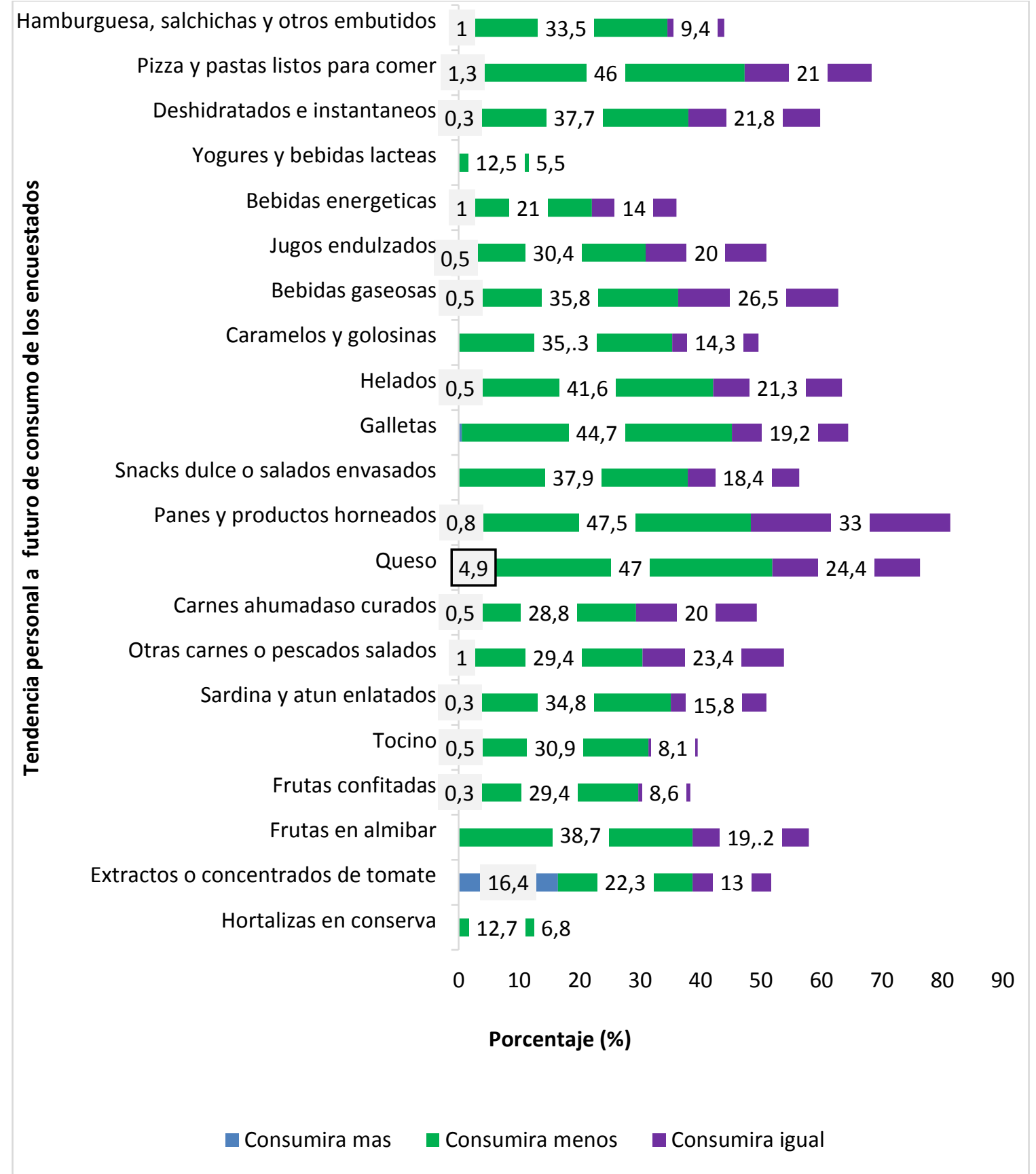

Nota. Adaptado de encuesta Alimentación saludable y las implicancias de la normativa en el consumidor-2019. 


\section{Figura 12}

Representación acerca la decisión de compra que tendrá el encuestado al ver los símbolos sujetos a la ley $N$ a 30021 en los empaques del producto.

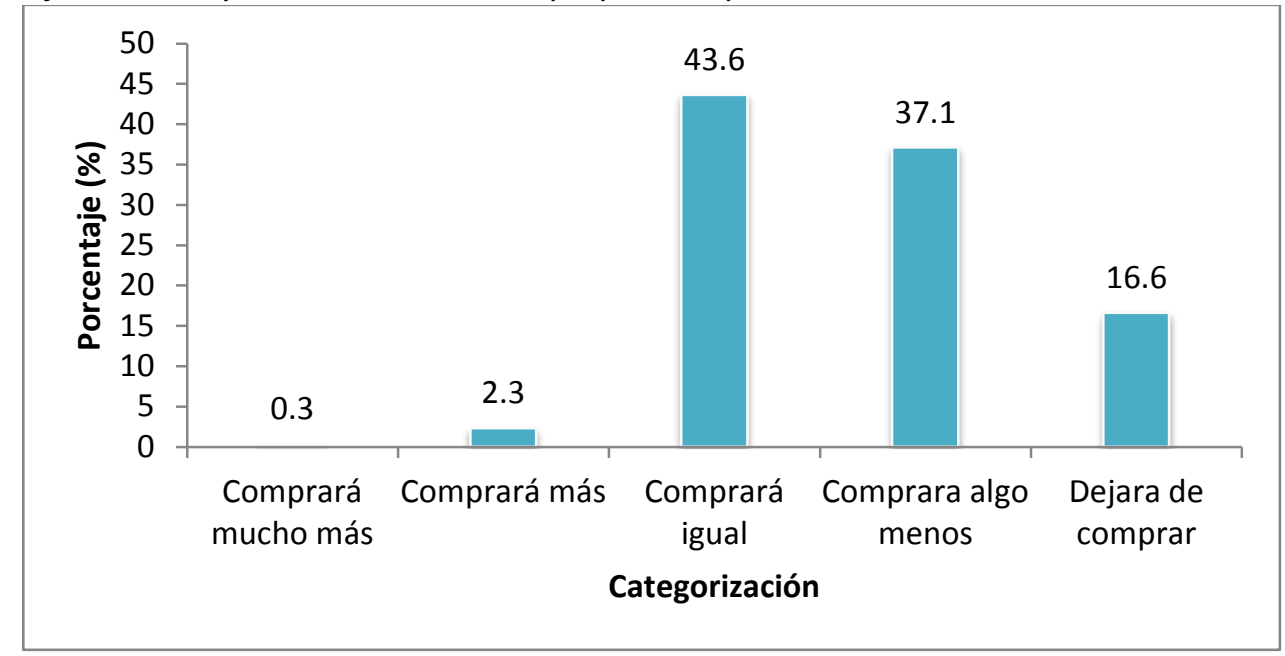

Nota. Adaptado de encuesta Alimentación saludable y las implicancias de la normativa en el consumidor - 2019.

\section{Figura 13}

Representación de la disponibilidad del encuestado a pagar por un incremento en el precio de productos con sistema de octágonos

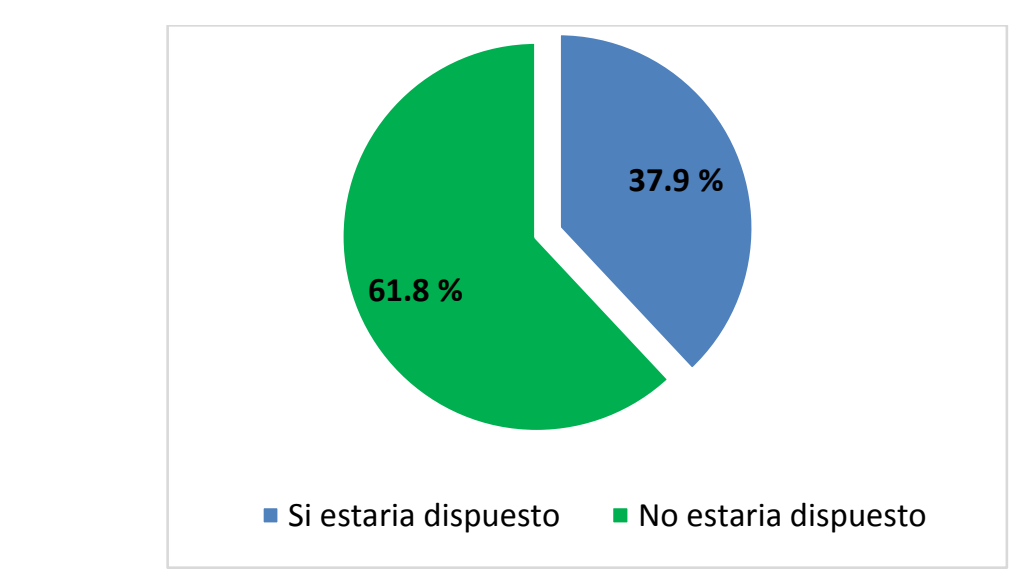

Nota. Adaptado de encuesta Alimentación saludable y las implicancias de la normativa en el consumidor - 2019.

4.9 Relación entre decisión de compra por parte del consumidor al agregado de nutrientes que mejoren la calidad del producto

Tal como se puede apreciar en la figura 14 , el $42,8 \%$ No estaría dispuesto a pagar un incremento de dinero por el agregado de nutrientes como las vitaminas, proteínas, carbohidratos y otros que 
mejoren, beneficien y aumenten la calidad del producto, Sin embargo el 45,2 \% afirma que Si estaría dispuesto a pagar un incremento de dinero por ese agregado, dicha información fue contrastado mediante prueba de chi cuadrado determinándose que existe una relación significativa $(p<0,005)$.

\section{Figura 14}

Representación del porcentaje adicional que estaría dispuesto a pagar el encuestado por el agregado de nutrientes que mejoren la calidad del producto.

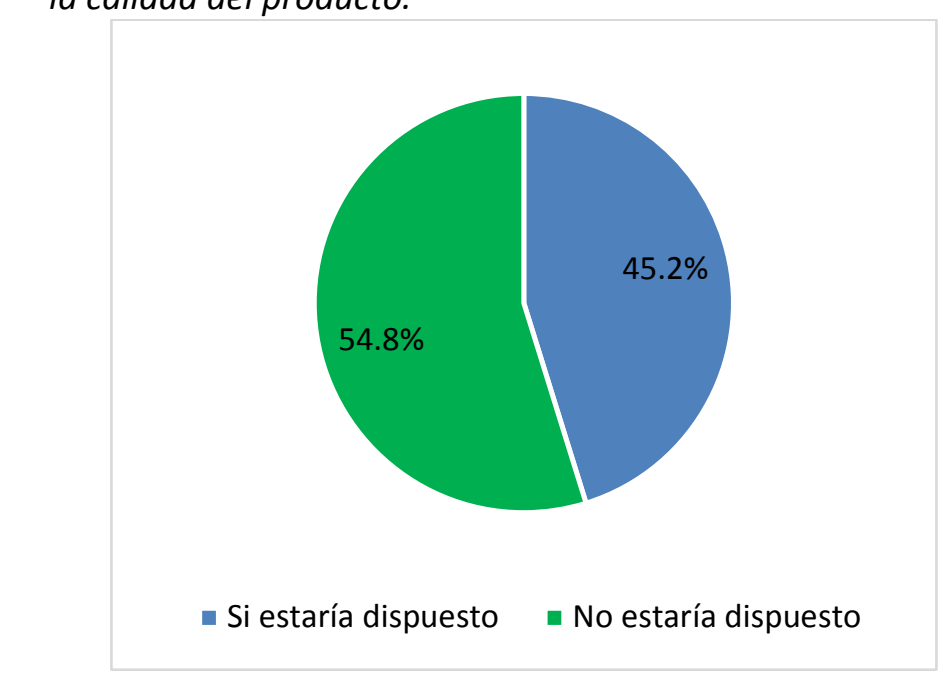

Nota. Adaptado de encuesta Alimentación saludable y las implicancias de la normativa en el consumidor - 2019.

\section{Discusiones}

Para conocer los alimentos y bebidas que son parte del día a día de la población tacneña, se ha elaborado un listado con productos procesados que según OMS (2014), los productos y bebidas procesadas son formulaciones listas para comer y beber; se basan en sustancias refinadas, con una cuidadosa combinación de azúcar, sal, grasa, y varios aditivitos. Incluye este listado bebidas azucaradas, snacks y comidas rápidas.

En un artículo periodístico recogido por el Diario el Correo (2019), el presidente de la Asociación Peruana de Emprendedores de la Panadería y Pastelería (ASPEN) Sr Pio Pantoja Soto informó que en Perú cada habitante consume un promedio de $35 \mathrm{Kg}$ de pan al año, cifra por debajo del promedio de otros países comparado con el consumo per cápita en Chile que es del $95 \mathrm{Kg}$ al año o en Argentina y Uruguay que es de $75 \mathrm{Kg}$ al año. Según los resultados los panes y productos horneados, son los productos procesados que más consumen los encuestados ya que es el acompañante tradicional del desayuno, almuerzo y cena, por otro el queso es el producto lácteo más consumido y en la lista general el segundo más consumido, al respecto, en el Perú, según Calle \& Solano (2004) el consumo de queso fresco es mayor al consumo de quesos maduros, esto debido a su bajo costo y usos variados. El otro aspecto a considerar resulta ser la comercialización de productos lácteos sin declarar su verdadera naturaleza en el etiquetado y carencia de idoneidad, decidiendo que el consumidor ingiera un producto que dista mucho de ser el que supone está adquiriendo. 
Debe mencionarse que a estos fines es importante resaltar que el consumidor promedio es una persona que no está acostumbrada a analizar el contenido del etiquetado. El portal de ESAN (2018) informó que a través de una encuesta realizada por IPSOS Perú por encargo de la Dirección de la Autoridad Nacional de Protección al Consumidor en el 2015, reveló que solo una cuarta parte de los encuestados lee las etiquetas de los productos. Comparando con los resultados de la encuesta realizada en la ciudad de Tacna resulta que solo el diez por ciento de los encuestados siempre lee las etiquetas. Mientras que una cuarta parte nunca lee las etiquetas. Como la mayoría de personas no lee las etiquetas no hay estudios referenciales con los cuales se pueda contrastar los resultados en este aspecto. Todo producto lleva en su etiqueta información respecto al mismo resaltando nombre, condición, fecha de elaboración, fecha vencimiento, contenido, valor nutricional e instrucciones. Adicionalmente el comerciante agrega a estas el valor económico del mismo.

La decisión del consumidor con relación a la información y atributos del productos, considerando el poco tiempo e interés en analizar esta, varía de acuerdo a la persona que lo adquiere a sus costumbres e idiosincrasia, lo cual se evidencia en el hecho de que preguntados los encuestados más de un tercio de los mismo reconocieron en el precio la principal característica que tienen en cuenta al momento de adquirir un producto procesado.

El estudio elaborado en la ciudad de Tacna en el año 2019 revela que casi la mitad de los encuestados si tenía conocimiento de la Ley de Alimentación Saludable. La diferencia que se puede apreciar es que luego de un año de difusión y debate público el conocimiento sobre dicha ley se ha incrementado, debido a la publicidad indicada así como por el hecho de que diversas empresas ya han empezado a implementar el sistema octagonal en productos procesados pese a que su obligatoriedad rige a partir del 17 de junio del año en curso. En este proceso de difusión de la ley la mayoría de los peruanos encuestados según DATUM INTERNACIONAL están de acuerdo en que los alimentos envasados contengan un rotulo de advertencia en sus empaques sobre los niveles de sodio, azúcar y grasas. De la misma manera la mayorías de encuestados en la ciudad de Tacna coinciden en estar de acuerdo en que los alimentos procesados contengan un rotulo de advertencia, ya que si bien es complicado entender el valor nutricional y los porcentajes de los distintos nutrientes, la figura de "alto en" despierta cierta impresión en personas de todas las edades, principalmente en niños y jóvenes, que al estar en busca de una respuesta de un adulto, este le tendrá que explicar el riesgo de consumir ese alimento y mucho más en exceso del mismo.

Lo manifestado evidencia tanto a nivel regional como nacional que los consumidores empiezan a demanda mayor información clara y coincida respecto de los características de los nutrientes críticos de los alimentos, de manera tal que le permitan tomar una decisión respecto de los alimentos que ingerirá y sus eventuales implicancias en la salud.

Es claro que los consumidores no pueden tener una valoración certera delo que no conocen y por ende la valoración de la alerta alimentaria se realizara en función a su conocimiento y su propia realidad. Según el estudio realizado por AXER Consultores (2019), realizado del 19 al 22 de marzo del 2019 algunas personas consideran que hay sellos más importantes que otros. Los octágonos más importantes para la población limeña encuestada (población de 19 a 59 años de edad) son "alto en azúcar" y "alto en grasas saturadas". Paralelamente en el estudio realizado a la población tacneña resulta que los octágonos más importantes son los que advierten "alto en azúcar" y "alto en grasas saturadas". Esto evidencia que existe un conocimiento más claro de los perjuicios que puede ocasionar el consumo elevado de azúcar más aun cuando es un producto de consumo diario, a diferencia de las grasas trans que población o no las conoce o sabe cómo definirlas pese a que en muchos casos está incluida en su dieta diaria. En este mismo orden de ideas, debemos analizar si el etiquetado es lo 
suficientemente claro y entendible, ya que compra de los productos se realiza en un corto periodo de tiempo que permite mayor estudio del contenido nutricional.

Analizando lo expuesto frente a los resultados del estudio realzado en la ciudad de Tacna, resulta que casi la mitad de la población tacneña encuestada afirma que mejor entiende el sistema de octágonos.

Estimaciones del Ministerio de Salud, que el $71 \%$ de las galletas que se comercializan en el país deberían etiquetarse con "alto en grasas saturadas"; el 62\% de snacks salados, con "alto en sodio"; el $54 \%$ de las bebidas lácteas con "alto en azúcar"; el $48 \%$ de los cereales para el desayuno con "alto en azúcar". La mayoría de las gaseosas y jugos en caja deberían también etiquetarse. De acuerdo a las encuestas realizadas en la Región de Tacna los snacks dulces o salados son relacionados con el octágono "alto en azucares". También los caramelos, golosinas, helados y galletas son relacionados con el mismo octágono "alto en azucares".

La asociación que hace el consumidor con este octágono se debe a que manifiestamente se trata de productos dulces, pero no lo asocia a otros octágonos como ser "alto en grasas trans" o "alto en grasas saturadas" tal vez por desconocimiento o por el hecho de que las grasas no tienen un sabor predomínate en el producto. Los encuestado de la región Tacna, asocian los alimentos instantáneos al octágono "alto en azúcar" y "alto en sodio" Asociado mayormente por el sabor del producto ya elaborado. Aportan agua y sales minerales que es lo que se debe reponer tras el desgaste físico, pero estas además contienen una gran cantidad de azúcar desnaturalizando su finalidad principal. Es relacionado por los encuestados con el octágono "alto en azucares", el yogur es un producto altamente calórico contiene normalmente mucha grasa que es la que le da consistencia cremosa, su sabor originalmente es agrio y la industria lo soluciona añadiéndole azúcar y además mermelada o incluso cereal endulzado, miel, frutas en conserva o granola lo que hace que deje de ser saludable.

Se debe de tener en cuenta que la mayoría de los productos procesados críticos son asociados principalmente con los octágonos "alto en azúcar" y "alto en sodio" .ningún producto tuvo como mayoría los octágonos "alto en grasas trans" o "alto en grasas saturadas" pese a que en muchos de los productos estos son sus principales componentes.

El origen de muchas enfermedades no se encuentra siempre en deficiencia congénita en los órganos del cuerpo humano, sino que existen factores extrínsecos que deciden su aparición, uno de estos factores es la alimentación.

Se ha podido determinar que el sobre peso y la obesidad conllevan a enfermedades como las cardiovasculares que incluye diabetes, presión alta, colesterol alto, entre otras. En el Perú las mayores prevalencias de sobre peso y obesidad se han encontrado en las regiones de Tacna y Moquegua (precisar fuente), Según la Direccion Regional de Salud Tacna (2017) más de seis mil personas padecen de diabetes en esta ciudad, que es una enfermedad silenciosa sin mayores síntomas en sus inicios, pero de lamentables consecuencias como ser hiperglucemia, problemas visuales llegando inclusive a la perdida dela visión, desarrollo de ulceras en pies y otras partes de la piel de tal magnitud que en algunos casos conlleva la amputación de partes o la totalidad de las extremidades inferiores.

Si la diabetes y enfermedades asociadas son de origen metabólico y las personas no tiene reparo en la ingesta de alimentos procesados altos en azucares, sales, grasas saturadas y trans directamente están originando la agudización de las enfermedades y el aumento de las personas afectadas por estas enfermedades no transmisibles. Entonces podemos colegir que la salud y las enfermedades guardan una relación directa entre sí con el tipo de alimentación que consuman, por eso actualmente el problema de 
obesidad y sobrepeso son problemas de salud pública pese a lo cual en Perú pese a los esfuerzos viene incrementándose diariamente.

De acuerdo con las recomendaciones de la Asociación Americana de Diabetes, la terapia nutricional es muy importante en la prevención y tratamiento de la Diabetes, con el objeto de controlar los niveles de glucosa en sangre, normalizar los niveles de presión arterial, evitar el aumento de peso y las complicaciones de la enfermedad. Las especialistas en nutrición Durán Agüero, E. Carrasco Piña y M. Araya Pérez (2012) recomendaciones de cantidad y calidad de los hidratos de carbono deben ser realizados buscando siempre los beneficios generales del uso del índice glicémico y la carga glicémica. Las dietas con bajos carbohidratos se utilizan para mejorar el perfil glicémico.

Según la investigación de Araya (2017), los resultados de su investigación muestran que la ley genera una disminución en el gasto en productos con etiqueta que varía aproximadamente entre $15 \%$ y $31 \%$ en chocolates-golosinas, snacks y bebidas, esto se debe a que por sí ya se sabe de qué estás poseen altas cantidad de nutrientes críticos, pero al momento de comprar, las personas más se guían por los antojos y necesidades, es por eso que cuando se vean los etiquetados, hará reflexionar rápidamente de las consecuencias de tal.

En jugos, las etiquetas impuestas por la ley provocan una disminución del consumo de productos etiquetados entre un $17 \%$ y $60 \%$. Para Araya, otro lado ver los productos etiquetados al momento de la compra provoca un mayor cambio de conducta que solo la percepción de que un producto es no saludable, esto quiere decir que el consumidor no dejará de pagar, solamente buscará algún

En términos generales los encuestados de la ciudad de Tacna manifiestan que respecto de los productos críticos procesados su consumo será menor. Esta disminución en las compras decidirá una baja en las ventas de las empresas con el consiguiente desmedro económico. Esto obligara a las empresas a auto regularse y mejorar la formulación de sus productos ya que el consumidor estará mejor informado y su selección será más consciente llevándolo a optar por un alimento más saludable.

La toma de decisiones es un proceso personal y que se asume generalmente en breves segundo, esto nos lleva a la conclusión que las consecuencias de las decisiones son exclusivamente personales. Es en este punto que el Estado busca que el consumidor tome una decisión informada al momento de decidir y que asuma conciencia de las implicancias que va a tener en su salud.

Los resultados de la encuesta muestran que el sistema de octágonos influencia en la decisión de compra y consecuente consumo. Pero paradójicamente la mayoría de consumidores refiere comprar que comprar igual, esto puede deberse a que la encuesta se ha trabajado sin que los encuestados vean en el mercado productos con etiquetado, consecuentemente no conocen del impacto que conlleva el etiquetado. También es posible que desconozcan los componentes de algunos de los productos procesados a los cuales consideraba saludables. $Y$ también es posible que no consuma mayormente productos procesados o que no presente mayor interés sobre el tema.

Lo ideal sería que ante la información brindada el consumidor asuma conciencia de los riesgos en el consumo de los alimentos procesados y que ello conlleve a modificar su decisión, de manera tal que el consumo de estos productos disminuya o se suspenda, que lo que resulta ser el objetivo final de la Ley de Alimentación Saludable.

Cabe resaltar que si bien en Chile esta ley tiene dos año de implementada, según el análisis de la Universidad Diego Portales (2016) se ha evaluado los resultados obtenidos en este periodo, llegando a la 
conclusión de que la venta de productos ha disminuido, lo que significa que el consumidor ha evaluado y valorado la información brindada en los octágonos. Queda claro que en esta primera etapa como consecuencia de la ley de octágonos, para Huerta (2018) los consumidores han variado su conducta al adquirir productos particularmente en niños, pero también se ha dado en la industria un cambio de actitud en el sentido de implementar la disminución significativa del contenido promedio de azucares en bebidas azucaradas, lácteos y cereales de desayuno, además de una reducción relevante de sodio en quesos y cecinas

Este análisis en nuestro país aún no es posible realizar hasta la entrada en vigencia de la ley, pero se esperar obtener similares resultados en la conducta del consumidor y en la actitud de los empresarios en la reformulación de los contenidos alimenticios.

Por otro lado, la población encuestada no está dispuesta a pagar un adicional en el precio del producto que cuente con un agregado de nutrientes pues si desease este incremento adquiriría uno específico a estos fines. Esta reacción de los encuestados es alarmante pero esperada, OMS (2017) resalta la carencia de nutrientes ha disminuido considerablemente en los alimentos en los últimos años, esto agrava la situación de desnutrición y genera un gran problema de salud pública, sobre todo en países con grandes fábricas de alimentos industrializados. Muchos encuestados se negaron a pagar más dinero por el aumento de nutrientes indicando que comprarían otro específico, pero la realidad es distinta, ya que al estar en busca de sabores determinados, es poco probable que el consumidor arriesgue su satisfacción al momento de comprar.

Los encuestados no analizan que el agregado de nutrientes tiene doble favorecimiento, el primero es que agrega nutrientes que probablemente consuma poco o haya dejado de consumir y el segundo es que puede seguir un patrón de consumo sin cambios tan radicales o sabores nuevos.

\section{Conclusión}

El sistema de alerta de octágonos permite mejorar el nivel de información de los consumidores respecto al tipo de nutrientes críticos que contiene los distintos alimentos, permitiéndole tomar una decisión informada. Las encuestas evidencian que es con motivo de la Ley de Alimentación Saludable, La población reconoce que su alimentación es alta en azucares, sodio y grasas y sus consecuencias se reflejan en el hecho de que Tacna es la ciudad con el índice más alto de sobrepeso y obesidad. La población tiene una actitud positiva frente a la aplicación al sistema de octágonos alimentarios de la Ley de Alimentación Saludable, debido a que es informativa, sencilla y fácil de entender, permitiéndole tomar una decisión consciente respecto a las implicancias en su alimentación y salud.

También considera que el azúcar es el nutriente crítico más perjudicial a la salud. Esto se debe al hecho de que el azúcar es un producto de uso cotidiano y conocido por la misma denominación, a diferencia de los otros nutrientes críticos que responden a otra denominación como es el caso del sodio o nutrientes que no son conocidos como es el caso de las grasas trans. 


\section{Referencias Bibliográficas}

Araya Aburto, S. A. (2017). Efectos del etiquetado de alimentos en el comportamiento de los consumidores.

AXER Consultores. (2019). Impacto de consumo de alimentos etiquetados con octógonos. Lima.

DATUM internacional. ((2018). Ley de alimentación saludable, Informe especial de opinión publica. Lima: OP 500-0118.

Durán Agüero, S., Carrasco Piña, E., \& Araya Pérez, M. (2012). Alimentación y diabetes. Nutrición Hospitalaria, 27(4), 1031-1036.

ESAN. (5 de Abril de 2018). Conexión ESAN. Obtenido de Conexión ESAN.

Gestión, D. (2019). El 63\% de consumidores reducirá consumo de alimentos etiquetados con octógonos.

González, R. (19 de junio de 2018). ¿Qué opina sobre el etiquetado en el Perú?

Hernández, A. (2016). Análisis comparativo de la NOM - 051 - SCFI/SSA1 -2010 y el sistema de etiquetado frontal nutrimental de otros países. Ciudad de México.

Huerta, E. (2018). ¿Cómo le fue a Chile con este etiquetado de alimentos procesados?

La Republica. (15 de junio de 2016). Larepublica.pe. Obtenido de Larepublica.pe. Leucemia, A. E. (s.f.). http://www.aeal.es/.

Correo, D. (15 de Marzo de 2019). Los peruanos consumen 35 kilos de pan al año, muy por debajo de otros países. El Correo.

OMS. (2014). Consumo de alimentos y bebidas ultra-procesados en. OMS. OMS. (11 de OCTUBRE de 2016). www.who.int.

OMS. (31 de AGOSTO de 2018). Alimentación sana. Obtenido de https://www.who.int. OPS. (JUNIO de 2018). OPS PERU. Obtenido de OPS PERU.

Organización Mundial de la Salud. (16 de Febrero de 2016). www.who.int. Obtenido de www.who.int.

Orjuela, R. (2017). ¿Qué es la comida chatarra? Bogotá: EDUCAR - CONSUMIDORES.

Pajuelo-Ramirez, J. (2015 de Abril de 2017). La obesidad en el Perú. La obesidad en el Perú, pág.7.

Peruano, G. (29 de febrero de 2019). Gob.pe. Obtenido de Gob.pe. Programa, N. D. (2018). Grasas: saturadas, insaturadas y tras.

La Republica, C. d. (2013). Ley 30021.

Salud, M. d. (2011). Dirección Nacional de Promoción de la Salud y Control de enfermedades no transmisibles. Obtenido de www.msal.gob.ar/ent/index.php/informacion-paraciudadanos/hipertensión-arterial 
Smith, P. (3 de Mayo de 2019). Economista detalla estudio sobre nuevo rotulado de alimentos procesados. (T. Perú, Entrevistador)

Tacna, D. R. (2017). Análisis de situación de salud. Tacna.

Universidad Diego Portales. (s.f.). Ley de Etiquetado: primeros efectos en la composición de alimentos y conductas. Santiago de Chile: 2016.

INEI. (2017). Censo Nacional 2017. Lima: INEI. 